\title{
Trivium
}

Revue franco-allemande de sciences humaines et sociales - Deutsch-französische Zeitschrift für Geistesund Sozialwissenschaften

33 | 2021

Concepts historiques fondamentaux - Démocratie

\section{Transmission et réception au Moyen Âge}

\section{Hans Leo Reimann}

Traducteur : Anthony Andurand

\section{(2) OpenEdition}

\section{Journals}

Édition électronique

URL : https://journals.openedition.org/trivium/7625

DOI : $10.4000 /$ trivium. 7625

ISSN : 1963-1820

Éditeur

Les éditions de la Maison des sciences de l'Homme

\section{Référence électronique}

Hans Leo Reimann, «II. Transmission et réception au Moyen Âge », Trivium [En ligne], 33 | 2021, mis en ligne le 07 juin 2021, consulté le 10 juin 2021. URL : http://journals.openedition.org/trivium/7625 ;

DOI : https://doi.org/10.4000/trivium.7625

Ce document a été généré automatiquement le 10 juin 2021

\section{(c) (i) () $\Theta$}

Les contenus des la revue Trivium sont mis à disposition selon les termes de la Licence Creative Commons Attribution - Pas d'Utilisation Commerciale - Pas de Modification 4.0 International. 


\title{
II. Transmission et réception au Moyen Âge
}

\author{
Hans Leo Reimann
}

Traduction : Anthony Andurand

\section{NOTE DE L'ÉDITEUR}

\section{p. 835-839 de l'article original}

1 [835] Au Moyen Âge, la notion de « démocratie » ne comptait pas parmi les concepts au moyen desquels on décrivait les rapports politiques ${ }^{99}$. On ne la rencontre pas dans les documents officiels, les actes, les rapports de droits et dans les enregistrements de faits juridiques ${ }^{100}$. Ce n'est que dans le sillage de la réception d'Aristote que le concept, comme topos de la littérature ayant trait à la philosophie du droit, fit son entrée dans le vocabulaire des érudits médiévaux. Dans la majorité des cas, le tableau des formations constitutionnelles et de leurs variétés déviantes proposé par Aristote dans la Politique a marqué la signification du terme de démocratie.

2 En se référant étroitement à Aristote, Thomas d'Aquin écrit: Si vero iniquum regimen exerceatur per multos, democratia nuncupatur, id est potentatus populi, quando scilicet populus plebeiorum per potentiam multitudinis opprimet divites. Sic enim populus totus erit quasi unus tyrannus ${ }^{101}$. Dans un autre contexte, le terme de " démocratie ", comme chez Aristote au demeurant, était repris comme une appellation générique pour désigner une forme de constitution, sans pour autant que le sens du terme fit écho à la domination corruptrice de la populace :

« Nam in politia democratia, in qua populus totus vult dominari, attenditur justum secundum quid, sed non simpliciter: ut scilicet quia omnes cives sunt aequales secundum quid, scilicet secundum libertatem, ideo habeantur aequales simpliciter, unde ea quae secundum legem democraticam statuuntur non sunt simpliciter, justa, sed aliqualiter ${ }^{102}$. » 
Thomas reconnaissait que certains éléments constitutionnels démocratiques [836] étaient conformes à l'ordre divin de la politia commixta, tout comme des éléments provenant d'autres formes constitutionnelles :

«Talis enim est optima politia, bene commixta ex regno, in quantum unus praeest; et aristocratia, in quantum multi principantur secundum virtutem; et ex democratia, id est potestate populi, in quantum ex popularibus possunt eligi principes, et ad populum pertinet electio principum. Et hoc fuit institutum secundum legem divinam ${ }^{103}$."

3 C'est chez Guillaume de Moerbeke, l'un des plus célèbres traducteurs d'Aristote, qui découvrit le philosophe grec à la faveur de sa collaboration avec Thomas d'Aquin, qu'apparaît pour la première fois la conception de la démocratie empruntée à Aristote ${ }^{104}$.

4 Dans l'espace francophone, on rencontre dans la seconde moitié du XIV siècle le concept de démocratie emprunté à la théorie aristotélicienne chez Nicolas Oresme, un autre traducteur d'Aristote: "Democratie est une espece de policie, en laquelle la multitude de populaire tient le princey ${ }^{105}$.» On trouve aussi chez lui le verbe "démocratiser", dont la signification est précisée de la manière suivante: "Les demagoges qui sont maintenant et qui veullent estre gracieulx au peuple democratizent moult de choses par les pretoires ou par les cours, c'est a dire que ils font et ordonnent trop de choses au plaisir du menu communet par flaterie ${ }^{106}$. »

5 Le sens péjoratif du terme "démocratie » se manifeste dans le Vocabularius optimus daté du milieu du XIV e siècle. Dans la section "De dignitatibus secularibus ", la demogracia, en tant que "pouvoir licencieux de la multitude» (gebufels fraventlicher gewalt), et la tymogracia, en tant que "pouvoir vertueux de la multitude» (gebufels tugendlicher gewalt), sont mises en opposition ${ }^{107}$.

6 Parmi les autres auteurs qui utilisèrent le concept de « démocratie » dans la description et la discussion des formes de constitutions, il convient de mentionner Marsile de Padoue et Engelbert d'Admont. Marsile de Padoue se réfère explicitement à Aristote quand il écrit :

«Sunt autem principative partis seu principatuum genera duo, unum quidem bene temperatum, reliquum vero viciatum. Voco autem bene temperatum [...], in quo dominans principatur ad commune conferens secundum voluntatem subditorum; viciatum vero, quod ab hoc deficit. Horum rursum generum utrumque dividitur in tres species: primum quidem, temperatum scilicet, in regalem monarchiam, aristocraciam et policiam; reliquum vero, viciatum scilicet, in tres oppositas species dividitur, tyrampnicam monarchiam, oligarchiam et democraciam ${ }^{108}$. "

Afin de caractériser la démocratie, il écrit :

«Policia vero, licet in una significacione sit commune quiddam ad omne genus vel speciem regiminis seu principatus, in una tamen ipsius significacione importat speciem quandam principatus temperati, in quo civis quilibet participat aliqualiter principatu vel consiliativo vicissim iuxta gradum et facultatem seu condicionem ipsius, ad commune eciam conferens et civium voluntatem sive consensum. Democracia vero illi opposita est principatus, in quo vulgus seu egenorum multitudo [837] statuit principatum et regit sola, preter reliquorum civium voluntatem sive consensum, nec simpliciter ad commune conferens secundum proporcionem convenientum ${ }^{109}$. »

7 Marsile voyait donc lui dans la démocratie une version corrompue de la politie au sens strict du terme $e^{110}$. 
8 Afin d'illustrer toute la difficulté de présenter la «théorie médiévale » relative à l'une des formes de constitutions, nous pouvons encore mentionner Engelbert d'Admont, également appelé Engelbert de Volkersdorf. Il attribue à Aristote le regroupement des formes de constitutions selon trois catégories: «regnum», "démocratie» et « olicratie ». En partant de cette lecture, dont il entend se démarquer, il parvient à une classification selon quatre couples, «regnum» et "tyrannie», "aristocratie» et " olicratie ", « olicratie » et " clerotis », « démocratie » et « barbarie », au sein desquels la seconde forme nommée est décrite dans chaque cas comme la version corrompue de la première. (L'« olicratie » se présente ainsi aussi bien comme une constitution droite que comme une constitution déviante.) Par « démocratie », Engelbert désigne la forme de domination à laquelle Aristote réservait strictement l'appellation de politeia : populus autem secundum legem et electionem seu consensum maioris partis dinffiiunt, [...] quorum est democratia ${ }^{111}$. Pour comprendre fidèlement le sens du terme "democratia» chez Engelbert, il conviendrait au préalable, à tout le moins, de déterminer de manière sûre ce qu'il entend, dans ce contexte, par populus, lex, electio et consensus maioris partis. Cependant, le fait, par exemple, que la procédure de la cooptation lors des «élections au conseil » [Ratswahl] dans certaines villes, ou encore le processus de l'« élection du roi » [Königserhebung] et les négociations préliminaires dont elle faisait l'objet pouvaient être désignées par le terme « electio » souligne toute la difficulté de traduire ces termes en allemand standard.

Pour comprendre un concept, comme celui de "démocratie», se référant à la communauté, la clarification qu'opère Engelbert de ces notions en renvoyant à l'image de la famille et du foyer est significative. Le chef de famille contrôlait sa femme de manière aristocratique, les enfants, de manière monarchique et les frères cadets de manière olicratique, cependant que les foyers présents dans le voisinage étaient selon lui unis les uns aux autres dans une forme démocratique ${ }^{112}$. Il est aisé de comprendre que le sens précis du terme « démocratique » [838], dans ce contexte, ne peut être saisi qu'en le replaçant dans la vision d'ensemble d'Engelbert et que cette conception de la démocratie que l'on saisit ici concrètement doit être assurément distinguée de celle d'autres auteurs écrivant à la même époque. Pour un concept comme celui-ci, on ne saurait fixer une signification uniforme, caractéristique et normalisée. Toute tentative en ce sens élude précisément les nuances importantes. Comme il n'existe guère de recherches spécialisées auxquelles il serait possible de se référer, toute définition claire de la notion de "démocratie» pour le Moyen Âge ne ferait qu'alimenter une impression erronée.

10 Ce que signifiait dans l'Antiquité la « démocratie » ou la "politie ", ce que signifiaient au Moyen Âge, à travers la reprise et le développement ou la transformation des concepts, la « res publica », la « res publica libera ", le «status popularis » et la « civitas popularis ", ce n'est pas à partir des mots, mais des choses elles-mêmes que l'on devra l'étudier ${ }^{13}$. Mais l'on parviendra ainsi, non à une clarification du concept de "démocratie", mais seulement à un déploiement des concepts de "respublica», « libertas » « status », « populus » et « civitas ».

11 Force est de constater que le concept de "démocratie », au Moyen Âge, ne fut utilisé, semble-t-il, que dans le contexte de la discussion issue de la littérature philosophique portant sur les formes de constitutions antiques que l'on connaissait. Ce n'est que bien plus tard que l'on entreprit d'utiliser le concept pour appréhender les conditions politiques concrètes. 

d'égalité et de codétermination que l'on pouvait déceler dans les sources de la constitution médiévale et aisément mettre en relation avec un tel concept. Ce n'est qu'au XIX ${ }^{\mathrm{e}}$ siècle que des tentatives furent mises en œuvre pour décrire, par exemple, les luttes menées par les guildes dans les villes ou les révoltes paysannes comme des phénomènes de démocratisation. Conformément à la compréhension médiévale du terme de "démocratie ", on ne saurait de toute façon s'attendre à ce qu'un groupe l'utilisât pour se décrire lui-même. Cependant, un groupement de guildes en lutte contre le conseil, par exemple, ou de paysans en lutte contre des seigneurs ne furent pas qualifiés de démocrates, même par leurs adversaires. La notion d'une égalité entre les membres d'un même ordre [Standesgleiche] qui émergeait au sein des états territoriaux, des corporations, des guildes et des communautés paysannes, ainsi que l'idée de codétermination, fondée sur un rapport de fidélité et se manifestant dans l'assistance et le conseil, avaient leur place dans le droit qui s'imposait à tous et dont tous acceptaient le caractère contraignant, selon le « bon vieil ordre ». Lorsqu'on luttait pour des droits de souveraineté [Herrschaft], on luttait pour son droit dans les limites de la constitution, de l'ordre en vigueur, et non pour que soit introduite une autre « forme de gouvernement ou de constitution ». Pour cette raison, on n'avait pas besoin des concepts érudits transmis ou reçus. Dans l'esprit de l'homme médiéval, le concept de " démocratie ", pour autant qu'il lui fût connu, [839] était défini comme la désignation d'une certaine forme de constitution dans l'Antiquité. Dans l'affrontement sur les droits et les devoirs des seigneurs, l'enjeu ne résidait pas, de fait, dans les formes de constitutions, mais dans le respect de la constitution, tenue pour l'expression du droit, contraignante pour tous et, il est vrai, faisant assez souvent l'objet d'interprétations divergentes. Il n'est donc pas étonnant que le concept de « démocratie » n'apparaisse pas dans les sources relatives aux ordres constitutionnels de l'Europe.

(Hans Leo Reimann)

\section{BIBLIOGRAPHIE}

Förster, F. (1853) : «Die Staatslehre des Mittelalters », Allgemeine Monatsschrift für Wissenschaft und Literatur, p. 832-863 et p. 922-936.

Fowler, George B. (1947) : Intellectual Interests of Engelbert of Admont, New York.

Fowler, George B. (1955) : « Engelbert of Admont's Tractatus de officiis et abusionibus eorum », in :

Essays in Medieval Life und Thought. Presentend in Honor of Austin Patterson Evans, New York, p. 109-122.

Fowler, George B. (1958) : « Engelbert of Admont and the Universal Idea », Fundamente, 3.

Godefroy, Frédéric (1898) : Dictionnaire de l'ancienne langue française, vol. IX, Paris.

Grimm, Jacob / Grimm, Wilhelm (1860) : Deutsches Wörterbuch, Leipzig. 
Marsile de Padoue (1968) : Le défenseur de la paix, traduction, introduction et commentaire par Jeannine Quillet, Paris.

Menzel, Ottokar (1941) : «Bemerkungen zur Staatslehre Engelberts von Admont und ihrer Wirkung », in : Corona Quernea, Festschrift Karl Strecker, Leipzig, p. 390-408.

Ors, Alvaro d' (1965) : « Vom antiken zum frühmittelalterlichen Staatsbegriff », Der Staat, 4, p. 360-368.

Posch, Andreas (1920) : Die staats- und kirchenpolitische Stellung Engelberts von Admont, Paderborn.

Scholz, Richard (1908) : « Marsilius von Padua und die Idee der Demokratie », Zeitschrift für Politik, 1, p. 61-94.

Segall, Hermann (1959) : Der « Defensor Pacis» des Marsilius von Padua. Grundfragen der Interpretation, Wiesbaden.

Suerbaum, Werner (1961) : Vom antiken zum frühmittelalterlichen Staatsbegriff. Über Verwendung und Bedeutung von Res Publica, Regnum, Imperium und Status von Cicero bis Jordanis, Munster.

Ueberweg, Friedrich (1928) : Grundriß der Geschichte der Philosophie, $11^{\mathrm{e}}$ éd., Berlin.

Wackernagel, Wilhelm (éd.) (1847) : Vocabularius optimus, Bâle.

\section{NOTES}

99. Il est significatif que la notion de «démocratie» ne soit pas traitée dans les Étymologies d'Isidore de Séville. Christian Gottlob Haltaus, Glossarium germanicum medii aevi (Leipzig, 1758) ne référence pas non plus le terme. Même le Mediae latinitatis lexicon minus (Jan Frederik Niermeyer [éd.], Leiden, 1954) n'a pas intégré le terme democratia.

100. On ne saurait pourtant exclure avec certitude que le mot «démocratie " apparaisse effectivement dans des passages isolés du groupe de sources mentionnées. Dans le cadre de ce travail, une multitude d'éditions de sources ont été à nouveau examinées, en particulier celles dans lesquelles on peut saisir des phénomènes souvent décrits au XIX siècle comme des processus de « démocratisation».

101. Thomas d'Aquin, De regimine principum, 16, in : Opera omnia, vol. XVI, Parme, 1865, p. 226 : "S'il y a un grand nombre d'hommes à la tête de ce mauvais gouvernement, il est appelé démocratie, c'est-à-dire pouvoir du peuple, ce qui arrive quand la populace opprime les riches au nom du peuple. De cette façon, tout le peuple ne fera qu'un tyran. » (trad. M. Védrine et al.).

102. Thomas d'Aquin, In decem libros ethicorum ad Nicomachum, in : Opera omnia, vol. V, p. 2 : « En effet, dans une constitution démocratique, en laquelle le peuple entier veut dominer, on s'attend à du juste sous un certain rapport, mais non strictement; c'est que, alors que tous les citoyens sont égaux sous un certain rapport, à savoir, sur le plan de la liberté, on les tient pour strictement égaux. Aussi, ce qui est statué d'après une loi démocratique n'est pas strictement juste, mais de quelque manière. » (trad. Y. Pelletier).

103. Thomas d'Aquin, Summa theologiae, II, 1, question 105, article 1, 5 : « Tel est le régime parfait, heureusement mélangé de monarchie par la prééminence d'un seul, d'aristocratie par la multiplicité de chefs vertueusement qualifiés, de démocratie enfin ou de pouvoir populaire du fait que de simples citoyens peuvent être choisis comme chefs, et que le choix des chefs appartient au peuple. » (trad. A-M. Roguet).

104. Cf. Ueberweg (1928), vol. II, p. 347 sq.

105. Nicolas Oresme, Motz estranges, cité in : Godefroy (1898), p. 300.

106. Nicolas Oresme, Politique, cité in : Godefroy (1898), p. 300. 
107. Wackernagel (1847), p. 38. Gefubels, qui se rattache à pófel, populus, doit être entendu au sens de « populace» (voir Grimm [1860], vol. II, p. 492, s. v. « Büffelvolk»).

108. Marsile de Padoue, Defensor pacis, I, 8, $2:$ «Il y a deux genres de parties gouvernantes ou gouvernements, l'un tempéré, l'autre corrompu. J’appelle le genre tempéré [...] celui dans lequel le prince gouverne pour le bien commun, en accord avec la volonté des sujets ; le corrompu, celui qui manque de ce caractère. En outre, l'un et l'autre se subdivisent en trois espèces: le gouvernement tempéré d'abord, en monarchie royale, aristocratie et république ; quant à l'autre, le corrompu, en tyrannie, oligarchie et démocratie.»

109. Ibid., I, 8, 3 : «La république, enfin, est, en un sens, quelque chose de commun à tout genre ou espèce de régime ou de gouvernement ; en un autre sens, cependant, elle désigne une certaine espèce de régime tempéré dans laquelle tout citoyen participe au gouvernement ou conseil selon son rang, ses moyens ou sa condition, pour le bien commun et en accord avec la volonté ou le consentement des citoyens. Mais la démocratie, son opposé, est un gouvernement dans lequel le bas peuple et la masse des pauvres établissent le gouvernement et gouvernent seuls, au mépris de la volonté des autres citoyens, et sans leur accord ; sans non plus tenir compte du bien commun dans une juste proportion. » (trad. J. Quillet ; Marsile [1968], p. 85-87).

110. La longue querelle liée à l'affirmation selon laquelle Marsile avait déjà formulé les notions modernes de souveraineté populaire et de démocratie montre combien il est aisé de mal comprendre cet auteur. Cf. Scholz (1908), p. 61 sq. Hermann Segall ([1959], en particulier p. 8 sq., p. 52-53 note 72, p. 63 sq.) a proposé une évaluation critique des résultats produits par la recherche jusqu'à ce jour.

111. Engelbert d'Admont, De regimine principum, I, 10 : « il s'agit d'une démocratie quand le peuple gouverne selon la loi et l'élection, resp. sur la base du consensus de la majeure partie », cité in : Posch (1920), p. 65, note 3. Cf. également Förster (1853), p. 832 sq. et p. 922 sq.

112. Sur la littérature dédiée, ainsi que sur la diffusion des écrits d'Engelbert, voir Menzel (1941), p. 390 sq. ; Fowler (1947) ; (1955), p. 109 sq. ; (1958).

113. Voir par exemple Cicéron, De Republica, I, 41-42: regnum - civitas optimatium arbitrio regi dicitur - civitas popularis [monarchie - gouvernement par les meilleurs ('optimates') gouvernement par le peuple] ; III, 48 : popularis res publica. Bien que le lecteur médiéval n'ait pas eu accès à ces écrits, on peut supposer que leur contenu était connu, notamment grâce à Augustin. Voir également Suerbaum (1961), ainsi que la recension très instructive d'Ors (1965), p. 360 .

\section{INDEX}

Schlüsselwörter : Demokratie, Mittelalter

Mots-clés : Démocratie, Moyen Âge

\section{AUTEURS}

\section{HANS LEO REIMANN}

Hans Leo Reimann était professeur à l'Université de l'Armée à Hambourg. Pour plus d'informations, voir la notice suivante. 